\title{
Dynamical symmetries and the Ermakov invariant
}

\author{
F. Haas* \\ Laboratório Nacional de Computação Científica - LNCC \\ Av. Getúlio Vargas, 333 \\ 25651-07 Petrópolis, RJ, Brazil \\ J. Goedert ${ }^{\dagger}$ \\ Centro de Ciências Exatas e Tecnológicas - UNISINOS \\ Av. Unisinos, 950 \\ 93022-000 São Leopoldo, RS, Brazil
}

\begin{abstract}
Ermakov systems possessing Noether point symmetry are identified among the Ermakov systems that derive from a Lagrangian formalism and, the Ermakov invariant is shown to result from an associated symmetry of dynamical character. The Ermakov invariant and the associated Noether invariant, are sufficient to reduce these systems to quadratures.
\end{abstract}

PACS number(s): 02.30.Hg, 02.90.+p, 03.20.+i

Keywords: Ermakov Systems, Lagrangian Formalism, Noether Symmetries, Reduction to Quadrature.

\footnotetext{
*ferhaas@lncc.br †'goedert@exatas.unisinos.br
} 


\section{Introduction}

Constants of motion (invariants or first integrals) are of central importance to study dynamical systems in general. This has motivated the development of several procedures by which constants of motion for dynamical systems (see [1] for a recent review) can be constructed. Among these, the application of Noether's theorem [2,3] deserves special attention in view of the physical appealing of the method. Indeed, Noether's theorem provides links between continuous symmetries of the action functional and constants of motion of the system. Among the classical results obtained by application of Noether's theorem are the conservation of energy, linear momentum and angular momentum. The associated symmetries correspond to the invariance of the action functional under time translation, space translation and space rotations, respectively.

Recently, Ermakov systems [4] were analyzed [5]-[8] via Noether's theorem. Ermakov systems, which are pairs of coupled, second-order ordinary differential equations, always admit at least one constant of motion, the Ermakov invariant. The work developed in [5]-[8] provided a consistent explanation for both the equations of motion and the invariant of Ermakov systems, as a result of an underlying Noether symmetry. The approach proposed, however, applied only to Ermakov systems where one of the equations decoupled from the other. This limitation has motivated comments [9] concerning the difficulty of deriving the Ermakov invariant from symmetry considerations only. A closer examination shows that references [5]-[8] assumed that one of the variables in the Ermakov system plays the central role, the other being regarded as a mere auxiliary variable. This point of view naturally led to systems where one of the equations (the so called auxiliary equation) decoupled from the other, of a more important character. In the present work, we consider the Ermakov systems as essentially coupled, with no dynamical variable playing any distinguished role. For this, we propose for the Ermakov systems not a one-dimensional but a two-dimensional Lagrangian formalism. Using this Lagrangian description, we search to construct coupled Ermakov systems for which Noether's theorem applies. In this context, both the Ermakov and all additional invariants - when they exist should result from the associated Noether symmetry which then provides a general framework to define, classify and solve Ermakov systems. We also remark that the use of a two-dimensional Lagrangian formalism for Ermakov 
systems has been suggested in a recent work [10].

A central issue with Ermakov systems concerns their complete integrability. Specificaly, we analyse the complete integrability of Ermakov systems in the Liouville sense [11], which, in turn, requires the existence of an underlying Hamiltonian structure. In fact, complete integrability requires, in this cases, the existence of at least a second invariant. The existence of Ermakov systems with a second invariant was first addressed by Goedert [12], who obtained a class of Ermakov systems possessing a second constant of motion. For Hamiltonian Ermakov systems, Liouville's theorem [11] warrants complete integrability if a second constant of motion exists in involution with the Ermakov invariant. Notice also that, strictly speaking, Liouville's theorem requires compact level surfaces of the constants of motion.

The present work focus on the application of Noether's theorem to Ermakov systems. The Lagrangian formalism constitutes the usual framework for the application of Noether's theorem [2] (and references therein). We thus need Ermakov systems that may be derived from a Lagrangian formalism. This has already been dealt with in the literature [13, 14]. Cerveró and Lejarreta [13] showed that conventional Hamiltonian Ermakov systems are completely integrable. By conventional Ermakov systems, we mean Ermakov systems having a frequency function depending on time only. However, as pointed out previously [15], a frequency function depending also on the dynamical variables does not destroy the main feature of Ermakov systems, namely the existence of the Ermakov invariant. This has motivated a number of studies [12], [14, 16, 17] on Ermakov systems with a generalized frequency function, depending on dynamical variables besides time. Following this trend, we do not restrict the frequency functions dependence on time only. Nevertheless we will show that the integrability of conventional Hamiltonian Ermakov systems results from a Noether symmetry, a fact that was not clear at all in the original reference [13]. Finally, we remark that dynamical symmetries have been considered in reference [18] for Ermakov systems of conventional type and not necessarily having Lagrangian character. Let us stress that in the present work the interest is centered on Noether symmetries, which may be assessed only when a variational description of the system is available. Besides, we do not restrict ourselves to conventional Ermakov systems.

The paper is organized as follows. In section 2, we obtain a class of Ermakov systems admitting a Lagrangian description, directly from the class 
of Hamiltonian Ermakov systems derived in reference [14]. In section 3 we show, by use of the converse of Noether's theorem [2], that the Ermakov invariant is the result of a Noether dynamical symmetry. Within the class of Lagrangian Ermakov systems, we also find the subclass of systems that possess Noether point symmetry. The Lagrangian Ermakov systems with Noether point symmetry, having a second constant of motion in involution with the Ermakov invariant, are Liouville integrable.

\section{Lagrangian Ermakov systems}

Ermakov systems are generally [14] defined as the system of equations

$$
\begin{aligned}
\ddot{x}+\omega^{2} x & =\frac{1}{y x^{2}} f(y / x), \\
\ddot{y}+\omega^{2} y & =\frac{1}{x y^{2}} g(x / y),
\end{aligned}
$$

where $f$ and $g$ are arbitrary functions of the indicated arguments and $\omega$ is an arbitrary function of time, the dynamical variables $x$ and $y$, and their time derivatives. For physical reasons, it is convenient to restrict the dependence to $\omega=\omega(t, x, y, \dot{x}, \dot{y})$. For brevity, we call $\omega$ the frequency function, even if it is not associated with any characteristic frequency of the system. We recall that, as shown in [14], only one arbitrary function of $y / x$ is sufficient to specify a generic Ermakov system if the frequency function is allowed to depend on the dynamical variables. However, for easier comparison with the results in the literature, we take (1-2) as our definition of Ermakov systems.

The central property of Ermakov systems is their Ermakov invariant,

$$
I=\frac{1}{2}(x \dot{y}-y \dot{x})^{2}+\int^{y / x} f(\lambda) d \lambda+\int^{x / y} g(\lambda) d \lambda .
$$

The Ermakov invariant is constant along the trajectories of (1-2) independently of the detailed dependence of $\omega$ on its arguments. The Ermakov invariant is most useful to construct a nonlinear superposition law [15] relating the solutions of (1) and (2). Here, however, we are interested in Ermakov invariants as one ingredient for the derivation of completely integrable Ermakov systems. 
Given the variational formulation, we can use some more specialized tools to investigate integrability, such as Noether's theorem [2]. This fact makes it interesting to consider not the general Ermakov systems (1-2), but only their subclasses that admit a variational formulation. In particular, we shall focus on Lagrangian descriptions, since it is on configuration space that Noether theorem is usually formulated [2].

We, therefore, pose the following question: under what conditions on the functions $\omega, f$ and $g$ does (1-2) possess a variational description? This question was partially answered by Cerveró and Lejarreta [13], who considered Ermakov systems with usual frequency functions, and by Haas and Goedert [14], in the case of frequency functions depending on dynamical variables. Both works adopted a variational description of the Hamiltonian type. Here, however, we are specially interested in Lagrangian descriptions and their connection with Noether's theorem. Therefore, we rewrite the results of reference [14] in terms of a Lagrangian formalism. Here this is the more direct approach, even if the usual procedure is to derive the Hamiltonian formalism from the Lagrangian description.

Using the results of [14] we may consider (1-2) as obtained from a Lagrangian function that represents the sum of a quadratic kinetic energy term and a potential energy term provided that the frequency function is taken in the form

$$
\omega^{2}=\frac{1}{R} \frac{\partial \bar{V}}{\partial R}(R, t)+\frac{\sigma(\theta)}{R^{4}},
$$

where $\sigma(\theta)$ is defined as

$$
\begin{aligned}
\sigma(\theta) & =\frac{(A \cos \theta+B \sin \theta)}{\psi^{2}(\theta) \sin \theta \cos ^{2} \theta} f(\tan \theta)+\frac{(B \cos \theta+C \sin \theta)}{\psi^{2}(\theta) \sin ^{2} \theta \cos \theta} g(\cot \theta) \\
& -2 \kappa\left(\int^{\tan \theta} f(\lambda) d \lambda+\int^{\cot \theta} g(\lambda) d \lambda\right),
\end{aligned}
$$

$f$ and $g$ being kept totally arbitrary. In $(4) \bar{V}(R, t)$ is an arbitrary function, and, $R$ and $\theta$ are defined by

$$
R^{2}=A x^{2}+2 B x y+C y^{2}, \quad \theta=\arctan (y / x),
$$

with $A, B, C$ and $\kappa$ numerical constants satisfying

$$
\kappa \equiv A C-B^{2} \neq 0
$$


Also, in (4) we used the notation

$$
\psi^{2}(\theta)=\left(A \cos ^{2} \theta+2 B \sin \theta \cos \theta+C \sin ^{2} \theta\right)^{-1} .
$$

As mentioned before, in general $\omega$ does not represent any physical frequency of the system, but we keep the nomenclature for convenience of notation. The associated Lagrangian function is then

$$
L=T-V,
$$

where the kinetic energy is

$$
T=\frac{1}{2}\left(A \dot{x}^{2}+2 B \dot{x} \dot{y}+C \dot{y}^{2}\right)
$$

and

$$
V=\bar{V}(R, t)+\frac{\kappa}{R^{2}}\left(\int^{\tan \theta} f(\lambda) d \lambda+\int^{\cot \theta} g(\lambda) d \lambda\right) .
$$

For the sake of completeness, we writte the corresponding Hamiltonian,

$$
H=\frac{1}{2 \kappa}\left(C p_{x}^{2}+2 B p_{x} p_{y}+A p_{y}^{2}\right)+V .
$$

An important point concerning Ermakov systems is their linearisation. As shown in [19], Ermakov systems with frequencies depending only on time are always reducible to a linear second-order ordinary differential equation. Latter on [20], the same linearisation was shown to apply to a subclass of Ermakov systems with frequency functions depending on dynamical variables. In the final part of the section, we verify in what cases the Lagrangian Ermakov systems just described may be linearisable following the procedure of references $[19,20]$. This procedure relies on the introduction of a new dependent variable $\varphi$ given by

$$
\varphi=\alpha(t) / R
$$

where $\alpha(t)$ is a function of time to be choosen conveniently. Also, we must use the polar angle $\theta$ as the new independent variable. The change of independent variable is acomplished by the relation

$$
\dot{\theta}=h(\theta ; I) / R^{2},
$$


which follows from the Ermakov invariant, where

$$
h(\theta ; I)=\frac{\sqrt{2}}{\psi^{2}(\theta)}\left(I-\int^{\tan \theta} f(\lambda) d \lambda+\int^{\cot \theta} g(\lambda) d \lambda\right)^{1 / 2} .
$$

Actually, in references $[19,20]$ the variables used are $\alpha(t) / r$ and $\theta$, where $(r, \theta)$ are the conventional polar coordinates, but here the choice (13-15) can be shown to be more effective.

Using the Lagrangian equations of motion obtained from (9) and the relations (13-15), we get

$$
h^{2} \frac{d^{2} \varphi}{d \theta^{2}}+h \frac{\partial h}{\partial \theta} \frac{d \varphi}{d \theta}+2 \kappa I \varphi=\alpha^{2}\left(\frac{\alpha \ddot{\alpha}}{\varphi^{3}}-\frac{\partial \chi}{\partial \varphi}\right),
$$

where $\chi$ is $\bar{V}$ expressed in terms of the new dependent variable,

$$
\chi=\chi(\varphi, t)=\bar{V}(\alpha / \varphi, t) .
$$

The left-hand side of eq. (16) is a linear form. Obviously, the complete equation will have a linear character if and only if the right-hand side of (16) is a linear form, which imposes

$$
\chi=\bar{\chi}(t)+\frac{a \varphi^{2}}{\alpha^{2}}-\frac{b \varphi}{\alpha^{2}}-\frac{\alpha \ddot{\alpha}}{2 \varphi^{2}},
$$

where $\bar{\chi}(t)$ is an arbitrary function of time which can be set to zero without loss of generality since it has no influence on the equations of motion. Moreover, $a, b$ are arbitrary numerical constants. The resulting linearisation reads

$$
h^{2} \frac{d^{2} \varphi}{d \theta^{2}}+h \frac{\partial h}{\partial \theta} \frac{d \varphi}{d \theta}+2(\kappa I+a) \varphi=b .
$$

The reconstruction of the solution of the original Ermakov system from the solution of (19) is made following the steps described in [19].

Here, the important point is that not all Lagrangian Ermakov systems are linearisable in terms of the proposed change of variables. Indeed, (18) imposes a particular dependence of the originally arbitrary function $\bar{V}$, namely

$$
\bar{V}(R, t)=\frac{a}{R^{2}}-\frac{b}{\alpha R}-\frac{\ddot{\alpha} R^{2}}{2 \alpha} .
$$

For $\alpha=$ cte., the resulting equations of motion can be shown to be in the class of Kepler-Ermakov systems [21]. For general $\alpha(t)$, we have a generalized time-dependent Kepler problem [22]. 


\section{$3 \quad$ Noether symmetries}

Let us consider the transformations

$$
\begin{aligned}
\bar{x} & =x+\varepsilon \eta_{1}(x, y, \dot{x}, \dot{y}, t), \\
\bar{y} & =y+\varepsilon \eta_{2}(x, y, \dot{x}, \dot{y}, t), \\
\bar{t} & =t+\varepsilon \tau(x, y, \dot{x}, \dot{y}, t),
\end{aligned}
$$

where $\varepsilon$ is an infinitesimal parameter and $\eta_{1}, \eta_{2}$ and $\tau$ are functions to be determined. As these functions may depend on the velocities, we are dealing with transformations of dynamical type. Dynamical symmetries may also contain a dependence of $\tau, \eta_{1}$ or $\eta_{2}$ on higher derivatives, but, for simplicity, we restrict the treatment to symmetries of form (21).

To proceed we introduce the following more convenient notation,

$$
\mathbf{q}=(x, y), \quad \eta=\left(\eta_{1}, \eta_{2}\right)
$$

and consider the action functional

$$
S[\mathbf{q}]=\int_{t_{0}}^{t_{1}} L d t
$$

Noether's theorem establishes a correspondence between continuous transformations that leave the action functional invariant (up to an additive numerical constant) and conservation laws. In particular, Noether's symmetry criterion [2] states that

$$
\tau \frac{\partial L}{\partial t}+\eta \cdot \frac{\partial L}{\partial \mathbf{q}}+(\dot{\eta}-\dot{\tau} \dot{\mathbf{q}}) \cdot \frac{\partial L}{\partial \dot{\mathbf{q}}}+\dot{\tau} L=\dot{\Lambda},
$$

for $\Lambda=\Lambda(\mathbf{q}, \dot{\mathbf{q}}, t)$ a function of the indicated arguments. The associated Noether invariant is

$$
J=\tau\left(\dot{\mathbf{q}} \cdot \frac{\partial L}{\partial \dot{\mathbf{q}}}-L\right)-\eta \cdot \frac{\partial L}{\partial \dot{\mathbf{q}}}+\Lambda .
$$

The Noether condition (24) may be used to determine simultaneously both the invariant and the action symmetry. However, if one invariant is known, we can always obtain an associated Noether symmetry, by use of the converse of Noether's theorem [2]. This usually leads to a symmetry of a 
dynamical character. In the present case, the Ermakov invariant is available, and the procedure may help to understand precisely its symmetry origin. Hence, we briefly review the converse of Noether's theorem, and apply it to our Lagrangian Ermakov system.

The converse of Noether's theorem [2], states that if $I(\mathbf{q}, \dot{\mathbf{q}}, t)$ is an invariant for a Lagrangian dynamical system with regular Lagrangian $L(\mathbf{q}, \dot{\mathbf{q}}, t)$, then it has the Noether symmetry (using component notation and summation convention)

$$
\begin{aligned}
\overline{q^{i}} & =q^{i}+\varepsilon\left(-g^{i j} \frac{\partial I}{\partial \dot{q}^{j}}+\tau \dot{q}^{i}\right), \\
\bar{t} & =t+\varepsilon \tau,
\end{aligned}
$$

where $\tau=\tau(\mathbf{q}, \dot{\mathbf{q}}, t)$ is an arbitrary function and $g^{i j}$ is the inverse of the Hessian matrix of the Lagrangian,

$$
g^{i j}=\left(\frac{\partial^{2} L}{\partial \dot{q}_{i} \partial \dot{q}_{j}}\right)^{-1} .
$$

Notice that the condition $\kappa \neq 0$ assures the existence of the inverse of the Hessian matrix.

The converse of Noether's theorem, as stated above, shows that all invariants for regular Lagrangian systems are derivable from a Noether symmetry which, generally, has non-point character. Hence, for regular Lagrangian Ermakov systems, the Ermakov invariant may be viewed as the result of a Noether symmetry. In the present case, using the Ermakov invariant (3), the Lagrangian (9) and equations (26-27), we find the following Noether symmetry associated to the Ermakov invariant $I$,

$$
\bar{t}=t+\varepsilon \tau, \quad \bar{R}=R+\varepsilon \tau \dot{R}, \quad \bar{\theta}=\theta+\varepsilon\left(-R^{2} \dot{\theta} / \kappa+\tau \dot{\theta}\right),
$$

where the transformation is expressed in terms of the coordinates $(R, \theta, t)$, and $\tau$ is a completely arbitrary function of time and the dynamical variables. The dynamical symmetry (29) gives directly the Ermakov invariant through Noether's theorem. Notice that it is not possible to use the freedom of choice of $\tau$ to reduce (29) to a point symmetry and we necessarily have a dynamical symmetry. Consequently the original studies [5]-[8] that considered only point symmetries in a one-dimensional Lagrangian formulation, were not 
apt to explain the Ermakov invariant in terms of Noether symmetries. The existence of a (two-dimensional) Lagrangian formalism pointed out in this work constitutes an essential ingredient. It is important to note, however, that not all Ermakov systems are Lagrangian and, hence the validity of the result is still restricted.

Noether's theorem may be useful also in the search for second invariants for Lagrangian Ermakov systems. With this goal in mind, we restrict the treatment to point transformations. In fact, a detailed calculation shows that allowing for dynamical symmetries depending at most on velocities does not gives additional new results, except for the derivation of the symmetry (29). But this symmetry is more easily derived by direct use of the converse of Noether's theorem. However, it is still possible that dynamical Noether symmetries depending on higher derivatives may yield new invariants for Lagrangian Ermakov systems.

Let us look for the existence of a second invariant for Lagrangian Ermakov systems. For this purpose, Cartesian coordinates $(x, y)$ are more appropriate then $(R, \theta)$ in the calculation of Noether symmetries. The Lagrangian (9), with the corresponding potential (11), becomes

$$
L=\frac{1}{2}\left(A \dot{x}^{2}+2 B \dot{x} \dot{y}+C \dot{y}^{2}\right)-\frac{\kappa}{R^{2}}\left(\int^{y / x} f(\lambda) d \lambda+\int^{x / y} g(\lambda) d \lambda\right)-\bar{V}(R, t),
$$

where $R=R(x, y)$ is given by $(6)$.

Not all Lagrangians of type (30) possess a Noether point symmetry. We, therefore, will search for the least restrictive conditions on functions $f, g$, $\bar{V}$ and on the parameters $A, B$ and $C$, compatible with the existence of Noether point symmetries. In such cases the Noether theorem yields a second invariant, independent of the Ermakov invariant.

The calculation of Noether point symmetries for a given Lagrangian is a well known procedure [3] and the main steps of this process can be summarized as follows. Inserting $L$ in the Noether criterion (24) results in a polynomial in the velocity components, which must be identically zero. The coefficients of the cubic terms imply that

$$
\tau=\rho^{2}(t),
$$

for any arbitrary function $\rho(t)$ of time only. This information and the coef- 
ficients of the quadratic terms lead to

$$
\begin{aligned}
& \eta_{1}=\rho \dot{\rho} x-W(t)(C y+B x)+a_{1}(t), \\
& \eta_{2}=\rho \dot{\rho} y+W(t)(A x+B y)+a_{2}(t),
\end{aligned}
$$

where $a_{1}, a_{2}$ and $W$ are arbitrary functions of time.

From the coefficients of the terms linear in $\dot{x}$ and $\dot{y}$, we get

$$
\begin{aligned}
& \partial \Lambda / \partial x=A \partial \eta_{1} / \partial t+B \partial \eta_{2} / \partial t \\
& \partial \Lambda / \partial y=C \partial \eta_{2} / \partial t+B \partial \eta_{1} / \partial t .
\end{aligned}
$$

These last two equations will have a solution $\Lambda$ provided that $\partial^{2} \Lambda / \partial x \partial y=$ $\partial^{2} \Lambda / \partial y \partial x$. This implies, after a simple calculation involving $\eta_{1}$ and $\eta_{2}$ given by $(32-33)$, that

$$
\dot{W}=0 .
$$

The corresponding solution for (34-35) is

$$
\Lambda=\Lambda_{0}(t)+\frac{1}{2}\left(\rho \ddot{\rho}+\dot{\rho}^{2}\right) R^{2}+\dot{a}_{1}(A x+B y)+\dot{a}_{2}(B x+C y),
$$

where $\Lambda_{0}(t)$ depends on time only. The remaining term, independent of velocity components in the Noether symmetry condition, yields a first order linear partial differential equation for the potential,

$$
\rho^{2} \frac{\partial V}{\partial t}+\eta \cdot \frac{\partial V}{\partial \mathbf{q}}=-2 \rho \dot{\rho} V-\frac{\partial \Lambda}{\partial t} .
$$

The potential $V$ must comply with the Ermakov form (11), and hence, is not completely arbitrary. Inserting $\eta_{1}, \eta_{2}, \Lambda$ and the Ermakov form for $V$, we get an equation for $\bar{V}$,

$$
\begin{gathered}
\rho^{2} \frac{\partial \bar{V}}{\partial t}+\rho \dot{\rho} \frac{\partial \bar{V}}{\partial R}+2 \rho \dot{\rho} \bar{V}= \\
-\dot{\Lambda}_{0}-\frac{1}{2}(\rho \ddot{\rho}+3 \dot{\rho} \ddot{\rho}) R^{2}-\ddot{a_{1}}(A x+B y)-\ddot{a}_{2}(B x+C y)+ \\
\frac{2 \kappa}{R^{4}}\left(a_{1}(A x+B y)+a_{2}(B x+C y)\right)\left(\int^{y / x} f(\lambda) d \lambda+\int^{x / y} g(\lambda) d \lambda\right)+
\end{gathered}
$$




$$
\kappa\left(\frac{a_{1} y-a_{2} x}{R^{2}}-W\right)\left(\frac{f}{x^{2}}-\frac{g}{y^{2}}\right) .
$$

Requiring $f$ and $g$ to remain arbitrary implies that the $f$ and $g$ dependent parts of the last equation vanish. This is obtained by choosing

$$
W=a_{1}=a_{2}=0 .
$$

Consequently,

$$
\rho^{2} \frac{\partial \bar{V}}{\partial t}+\rho \dot{\rho} \frac{\partial \bar{V}}{\partial R}+2 \rho \dot{\rho} \bar{V}=-\dot{\Lambda}_{0}-\frac{1}{2}(\rho \ddot{\rho}+3 \dot{\rho} \ddot{\rho}) R^{2} .
$$

The general solution of (41) is

$$
\bar{V}(R, t)=-\frac{\Lambda_{0}}{\rho^{2}}-\frac{\ddot{\rho}}{2 \rho} R^{2}+\frac{1}{\rho^{2}} U(R / \rho),
$$

where $U(R / \rho)$ is an arbitrary function of the indicated argument. In fact, we can take $\Lambda_{0}=0$ without any loss of generality since the addition of a function of time only in the Lagrangian will not affect the equations of motion. This completes the Noether point symmetry calculation.

We remark that (42) could have been obtained from the potential associated to the autonomous Hamiltonian Ermakov systems in [14] by performing a rescaling transformation $\tilde{x}=x / C(t), \tilde{y}=y / C(t), \tilde{t}=\int d t / C^{2}(t)$ and requiring the resulting equations to stay autonomous. However, Noether's theorem provides a more consistent basis for the reasons why the potential (42) is integrable: integrability here is viewed as resulting from a general symmetry consideration and not from an ad hoc transformation. Moreover, the calculation of Noether point symmetries provides a hint for the determination of the more general dynamical Noether symmetries.

Let us summarize our results so far. From Noether's symmetry criterion applied to the class of Lagrangian Ermakov systems of section 2, we obtained the symmetry transformations

$$
\bar{x}=x+\varepsilon \rho \dot{\rho} x, \quad \bar{y}=y+\varepsilon \rho \dot{\rho} y, \quad \bar{t}=t+\varepsilon \rho^{2} .
$$

The corresponding potentials can be expressed in terms of $f, g$ and another arbitrary function $U(R / \rho)$ as

$$
V=-\frac{\ddot{\rho} R^{2}}{2 \rho}+\frac{1}{\rho^{2}} U(R / \rho)+\frac{\kappa}{R^{2}}\left(\int^{y / x} f(\lambda) d \lambda+\int^{x / y} g(\lambda) d \lambda\right) .
$$


Consequently four arbitrary functions, namely $\rho(t), U(R / \rho), f(y / x)$ and $g(x / y)$, remain in the system. In $(R, \theta)$ coordinates, the Ermakov invariant and the invariant arising from point symmetry have the compact representation,

$$
\begin{aligned}
I & =\frac{1}{2} R^{4} \psi^{4}(\theta) \dot{\theta}^{2}+\int^{\tan \theta} f(\lambda) d \lambda+\int^{\cot \theta} g(\lambda) d \lambda, \\
J & =\frac{1}{2}(\rho \dot{R}-\dot{\rho} R)^{2}+U(R / \rho)+\kappa I(\rho / R)^{2} .
\end{aligned}
$$

For the sake of comparison, we writte, using configuration space variables, the corresponding non-constant, time-dependent Hamiltonian function which follows from (12),

$$
H=\frac{1}{2} \dot{R}^{2}+\frac{\kappa I}{R^{2}}-\frac{\ddot{\rho} R^{2}}{2 \rho}+\frac{1}{\rho^{2}} U(R / \rho) .
$$

An important point to check is the fact that $I$ and $J$ are in involution. This follows from the conventional definition of Poisson brackets and the relations $\dot{x}=\left(C p_{x}-B p_{y}\right) / \kappa, \dot{y}=\left(A p_{y}-B p_{x}\right) / \kappa$.

A simple inspection shows that level surfaces of the function $I$ are not compact, a fact that prevents the application of Liouville's theorem. Despite this, the exact solution for the Ermakov systems possessing the invariants (45-46) can be obtained as follows. For given $\rho(t)$, define new variables

$$
\bar{R}=R / \rho, \quad T=\int d t / \rho^{2}
$$

and transform the Noether invariant (45) into

$$
J=\frac{1}{2}(d \bar{R} / d T)^{2}+U(\bar{R})+\kappa I / \bar{R}^{2},
$$

an energy-like form. Using the energy method [11], we can find, by quadratures, $\bar{R}$ as a function of $T$, and then $R$ as a function of $t$. The exact solution is then obtained by inserting $R$ as a function of $t$ in the Ermakov invariant (45), which can then be interpreted as a first-order ordinary differential equation for the solution $\theta$. Being separable, this equation is again soluble by quadratures.

We observe that the Noether symmetry (43) is of the same type as the Lie point symmetry eventually admitted by Ermakov systems [23], as expected. 
Indeed, Noether point symmetries form a subgroup of the more general Lie point symmetry group of the same dynamical system [2]. At least in this case, however, the Noether symmetries result more interesting because they, directly, produce constants of motion.

In spite of the reduction of the Lagrangian Ermakov systems with Noether point symmetry to quadrature, these systems are not linearisable, in general. Indeed, using (20) and (42) we can show that the only two cases compatible with linearisation are

$$
U(\bar{R})=a / \bar{R}^{2}-b / \bar{R}
$$

or

$$
U(\bar{R})=a / \bar{R}^{2}+c \bar{R}^{2} / 2
$$

for $a, b$ and $c$ arbitrary numerical constants. Moreover, when $U$ has the form (50), the function $\alpha$ in (13) must be choosen as $\alpha=\rho$. When $U$ has the form (51), $\alpha$ must be choosen as any particular solution for the equation

$$
\ddot{\alpha}+\left(\frac{c}{\rho^{3}}-\ddot{\rho}\right) \frac{\alpha}{\rho}=0 .
$$

In all linearisable cases, the function $\rho(t)$ is left free.

We finally compare our result with other results in the literature. Grammaticos and Dorizzi have derived the potential (44) with $A=C=1$, $B=0$ and arbitrary $\rho(t)$ (see equation (60) of [24]) in their search for twodimensional systems with invariants quadratic in velocity. However, they did not recognize the Ermakov character of the system. Dhara and Lawande, in cases A) and C) in reference [25], found potentials that correspond to Ermakov systems or to Noether point symmetry. However, the possible intersection of the two classes of potentials, yielding Ermakov systems with Noether point symmetry, was not explored at all. Leach, Lewis and Sarlet [26] found a class of potentials with an invariant quadratic in velocity. Within this class, there exists an Ermakov subclass, a fact that was not pointed out precisely. The Ermakov structure in these systems was hidden due to the non usual character of the frequency function, depending on dynamical variables. Finally, the second invariant found by Goedert [12] is a Noether invariant which can be obtained in the present formalism, by choosing $A=C=0$, $B=1$ and $\rho=1$. 


\section{Conclusion}

In this paper we identified a subclass of Ermakov systems with two interesting properties, namely, of being Lagrangian and of possessing Noether symmetries of both point and dynamical character. We also show, by the converse of Noether's theorem, how the Ermakov invariant follows from a Noether symmetry of dynamical character. This is an alternative criterion to characterize and classify the Ermakov systems which should be considered parallel with the existence of Lie point symmetries [17, 23] or of special structure of the solutions on the complex plane [27]. Moreover, the class of Lagrangian Ermakov systems with Noether point symmetries presented in this paper may possibly be expanded further by relaxing our starting point in terms of the ansatz (9) for the Lagrangian. 


\section{Acknowledgments}

This work was partially supported by Fundação de Amparo à Pesquisa do Estado do Rio Grande do Sul (Fapergs) and Conselho Nacional de Desenvolvimento Científico e Tecnológico $(\mathrm{CNPq})$. The authors also acknowledge the Instituto de Física (IF-UFRGS) for continued support of their research projects.

\section{References}

[1] R. S. Kaushal, Int. J. Theor. Phys. 37 (1998) 1793.

[2] W. Sarlet and F. Cantrijn, SIAM Rev. 23 (1981) 467.

[3] F. Haas and J. Goedert, J. Phys. A: Math. Gen. 32 (1999) 6837.

[4] V. P. Ermakov, Univ. Isv. Kiev 20 (1880) 1.

[5] J. R. Ray and J. L. Reid, J. Math. Phys. 20 (1979) 2054.

[6] J. R. Ray, Prog. Theor. Phys. 65 (1981) 877.

[7] J. R. Ray, J. Phys. A: Math. Gen. 13 (1980) 1969.

[8] R. S. Kaushal and H. J. Korsch, J. Math. Phys. 22 (1981) 1904.

[9] H. Kanasugi and H. Okada, Prog. Theor. Phys. 93 (1995) 949.

[10] S. S. Simic, J. Phys. A: Math. Gen. 33 (2000) 5435.

[11] V. I. Arnold, Mathematical methods of classical mechanics. New York: Springer-Verlag, 1978.

[12] J. Goedert, Phys. Lett. A 136 (1989) 391.

[13] Cerveró and J. D. Lejarreta J. M. , Phys. Lett. A 156 (1991) 201.

[14] F. Haas and J. Goedert, J. Phys. A: Math. Gen. 29 (1996) 4083.

[15] J. L. Reid and J. R. Ray, J. Math. Phys. 21 (1980) 1583.

[16] K. Saermark, Phys. Lett. A 90 (1982) 5. 
[17] J. Goedert and F. Haas, Phys. Lett. A 239 (1998) 348.

[18] C. Athorne, J. Phys. A: Math. Gen. 31 (1998) 6605.

[19] C. Athorne, C. Rogers, U. Ramgulam and A. Osbaldestin, Phys. Lett. A 143 (1990) 207.

[20] F. Haas and J. Goedert, J. Phys. A: Math. Gen. 32 (1999) 2835.

[21] C. Athorne, J. Phys. A: Math. Gen. 24 (1991) L1385.

[22] A. Munier, J. R. Burgan, M. R. Feix and E. Fijalkow, Astron. Astrophys. 94 (1981) 373.

[23] P. G. L. Leach, Phys. Lett. A 158 (1991) 102.

[24] B. Grammaticos and B. Dorizzi, J. Math. Phys. 25 (1984) 2194.

[25] A. K. Dhara and S. V. Lawande, J. Math. Phys. 27 (1986) 1331.

[26] P. G. L. Leach, H. R. Lewis and W. Sarlet, J. Math. Phys. 25 (1984) 486.

[27] C. Athorne, J. Phys. A: Math. Gen. 24 (1991) 945. 